\title{
Téoros
}

Revue de recherche en tourisme

\section{Le tourisme de villégiature en Estrie}

\section{Roger Nadeau et Danielle Gilbert}

Volume 7, numéro 2, juillet 1988

Villégiature et tourisme

URI : https://id.erudit.org/iderudit/1080408ar

DOI : https://doi.org/10.7202/1080408ar

Aller au sommaire du numéro

Éditeur(s)

Université du Québec à Montréal

ISSN

0712-8657 (imprimé)

1923-2705 (numérique)

Découvrir la revue

Citer cet article

Nadeau, R. \& Gilbert, D. (1988). Le tourisme de villégiature en Estrie. Téoros, 7(2), 3-10. https://doi.org/10.7202/1080408ar d'utilisation que vous pouvez consulter en ligne.

https://apropos.erudit.org/fr/usagers/politique-dutilisation/ 


\section{Roger Nadeau* et Danielle Gilbert** en Estrie}

\section{Le tourisme de villégiature}
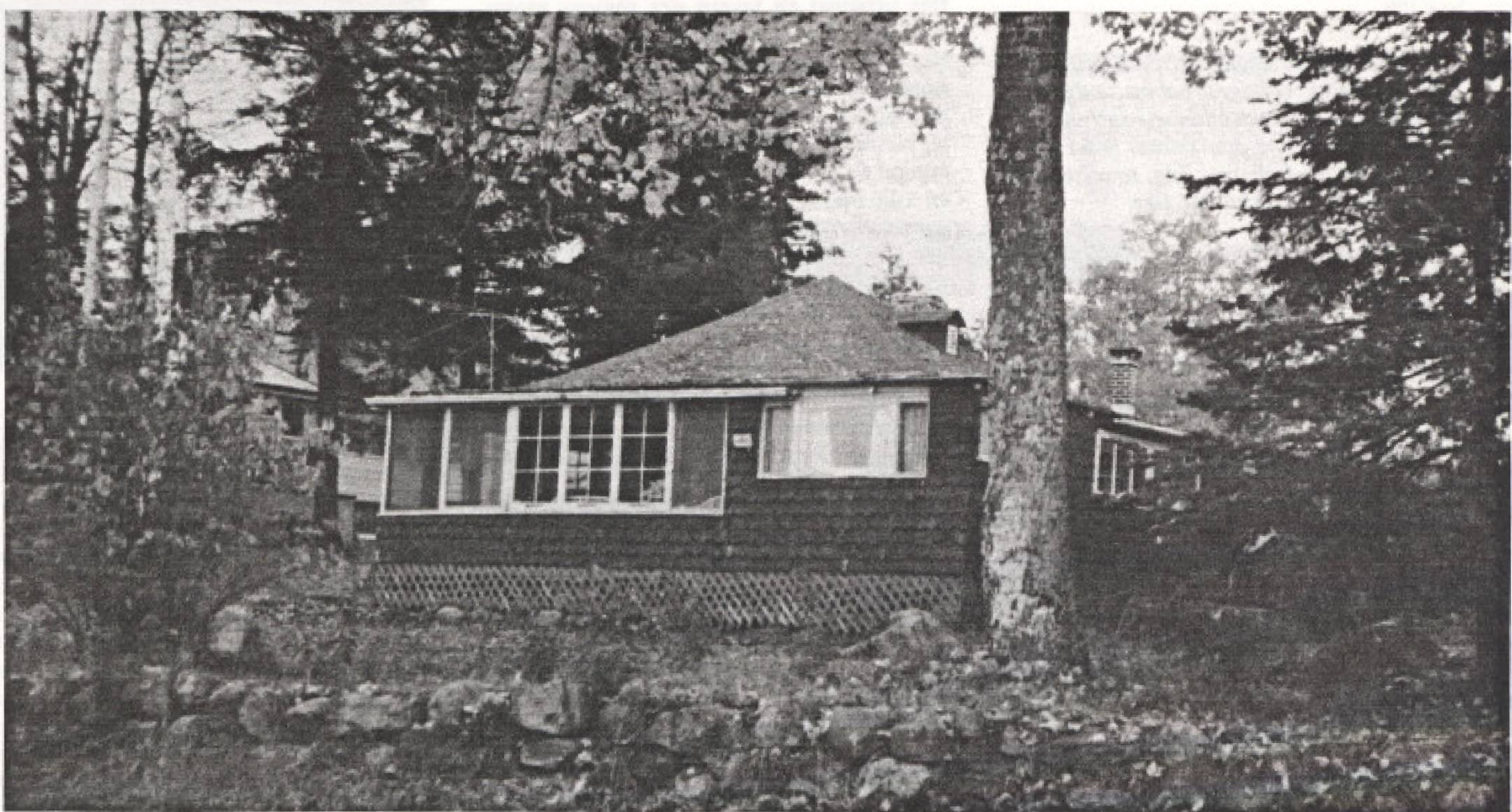

\section{Avant-propos}

Comme c'est souvent le cas dans le domaine du tourisme, il est habituellement souhaitable que les auteurs précisent au point de départ leur terminologie, la portée qu'ils conceddent à certains concepts ainsi que les philosophies qu'ils véhiculent. Ces points étant éclaircis, il sera sans doute plus facile pour les lecteurs de suivre le cheminement proposé.

\section{a) La villégiature}

Bien que le concept de villégiature soit assez large, il fait toujours référence à des formes de tourisme se pratiquant en relation avec le milieu naturel, la campagne, les zones littorales ou montagneuses. Ces secteurs sont recherchés comme antidote au rythme de vie trépidant imposé par nos sociétés postindustrielles caractérisées par les concentrations urbaines, par le stress, le bruit et la course soutenus. Quant cela lui est possible, l'individu cherche à fuir ce milieu agressant;

\footnotetext{
- Roger Nadeau est professeur au Département de géographie de PUniversité de Sherbrooke.

* Danielle Gilbert est detentrice d'une maituise en géographie de I'Universite de Sherbrooke.
}

les fins de semaine et la période des vacan= ces annuelles deviennent donc des moments privilégiés où il peut refaire le plein de calme, de détente et de décompression dans un milieu qui s'y prête. Ce besoin est devenu tellement criant que les zones lacustres situées à proximité des grands centres urbains ont littéralement été envahies par des masses de villégiateurs. Au Québec seulement, le nombre de résidences secondaires est passé de 138000 en 1971 à environ 325000 en $1984^{(1)}$. Ce phénomène n'est pas nouveau car dejà en 1963, Louis Burnet en signalait déjà les impacts majeurs sur nos modes de vie:

"Ce phenomène hebdomadaire et surtout annuel est d'une extrêne complexité. II représente la forme moderne du nomadisme, mais d'un nomadisme en perpétuelle évolution. Il transforme nos habitudes de vie et de pensée essentiellement sédentaires. Les notions de richesse, de densité de population. sont à réviser. Les grands courants humains se deplacent, les professions se diversifient, de nouvelles agglomérations naissent et l'économie s'adapte au nouveau concept: le semi-nomadisme des populations evoluées. "(2)
Étymologiquement, villégiature signifie "séjour à la campagne" et "maison de plaisance à la campagne" "(3). La villégiature est donc liée à la présence de ces maisons de plaisance (qu'on appelle maintenant davantage "chalet" ou "résidence secondaire") lesquelles constituent le mode d'hébergement du villégiateur séjournant à la campagne, dans la nature. Ce sont les qualités et les attraits particuliers de cette nature qui justifient et expliquent cette forme de tourisme ${ }^{(4)}$.

Mais bien sûr, le phénomène de la villégiature ne se limite pas strictement à la présence de milliers de chalets sur le pourtour des cours d'eau; il existe aussi, et de plus en plus, des centres de villegiature, des stations de villégiature, des condos en villégiature, des apart-hôtels de villégiature... etc. ... (5), Il est cependant utile de préciser que le présent texte ne porte que sur la réalité des résidences secondaires (chalets).

b) La résidence secondaire

Même si à priori le concept de la résidence secondaire semble facile à circonscrire, dans les faits, ce n'est pas toujours évident: 
" - Les résidences secondaires ne constituent pas un type net et précis mais elles forment plutôt un groupe arbitrairement identifie dans une continuité.

- Les caractéristiques de la résidence secondaire peuvent inclure d'autres modes $d$ 'hebergement tels les roulottes qui sont considérées par cerrains pays.

- La definition adopté affecte le nombre de residences secondaires identifies, et de ce fait, la géographie des résidences secondaires." "'(s)

"Second homes include vacation residences such as houses, cottages, cabins, lodges and residences for non-wacation use such as hotel rooms or suites, or apartments reserved as a convenience when traveling, to offset commuting problems, and the like." $n$

"Habitation appartenant à un membre du ménage et utilisée en saison comme lieu de villegiature ou pour les loisirs, par exemple. une cabane de chasse, un chalet de ski, un chalet d'eté ou n'importe quel logement servant surtout aux loisirs. Ne sont pas compris les roulottes, bateaux... "(8)

Comme on peut le constater à la lumière de ces dernières définitions, le phénomène de la résidence secondaire peut être appréhendé avec plus ou moins de latitude. Pour les fins du présent texte, la définition de la résidence secondaire a une porté assez restrictive: elle se limite à peu près strictement au chalet de plaisance implanté surtout sur le pourtour des lacs ou à flane de montagne:

"C'est la propriété personnelle d'une personne $n$ 'habitant pas la commune et d'origine généralement citadine, qui l'utilise généralement pour ses fins de semaine ou ses vacances, tout en pouvant la louer pour une certaine période. "w)

"C'est une habitation fixe, appartenant à un membre du ménage, utilisée périodiquement à des fins autres que le travail (détente et loisir surtout) et dont le ménage propriétaire sera habituellement le principal utilisateur. "wol

\section{La villégiature: la mal aimée de I'industrie touristique}

Si on reconnait que le tourisme se caractérise par un déplacement hors de son horizon habituel, par un séjour d'au moins une nuitée, et cela réalisé comme activité de loisir et de consommation, on doit bien admettre que la villégiature en résidence secondaire fait partie intégrante du phénomène touristique et qu'elle devrait ètre considérée comme tel. Il nous apparaît en effet que la villégiature est une des formes de tourisme qui apporte le plus à une région. Ce sont les villégiateurs qui, les premiers, identifient et délimitent les régions touristiques et c"est en s'appuyant sur leur présence importante que les autres types de services touristiques viennent s'implanter. Le thếtre de la Marjolaine s'est implanté à Eastman en comptant d'abord sur la clientèle des villégiateurs; il en fut de meme pour les relais gastronomiques, auberges de campagne et autres commerces ne pouvant se justifier par la population en résidence permanente.

La villégiature est un des plus importants facteurs contribuant à atténuer les disparités régionales sur le plan économique. Le tourisme de villégiature provoque, en effet, un déplacement salutaire des ressources économiques des régions riches (les grands centres urbains) en faveur des régions moins bien pourvues (les régions rurales périphériques aux zones urbaines). Une partie importante de cet impact s'explique par le dédoublement de services qu'implique la villégiature; le proprietaire de chalet s'est équipé de meubles et d'appareils ménagers en ville mais il doit en faire autant à la campagne; il paie des taxes scolaires et municipales en ville, et il fait de même à la campagne; en ville, il paie des assurances pour sa maison, achète des matériaux de construction, de la nourriture et des vêtements, assume des dépenses liées au chauffage, à l'entretien de l'automobile ainsi qu'à ses loisirs et divertissements... etc. ... et il en fait tout autant à la campagne, à sa résidence secondaire. Une partie appréciable de ces dépenses sont effectuées localement, a l'intérieur de la zone de villegiature. Si on se réfere à l'étude sur Magog-Orford dont il sera question ultérieurement (portant sur 4644 chalets), chaque proprićtaire de résidence secondaire a consacré, en moyenne, sur une base annuelle, $3900 \$$ en 1984 pour pouvoir profiter de son chalet; de cette somme, 2798\$, soit $71,7 \%$ étaient dépensés en région ${ }^{(11)}$. Il $n^{\dagger} y$ a pas beaucoup de forme de tourisme au Québec qui peuvent se vanter de générer un impact économique aussi important, surtout si on considère qu'il s'agit ici d'un "tourisme captif", c'est-à-dire que dû à la présence fixe du chalet et donc d'un investissement personnel majeur, le villégiateur est donc presque obligé d'y revenir année après année, ce qui fait que le $3900 \$$ se multiplie par $10,20,30$ ou 40 années... sans aucune subvention gouvernementale!

Cette activité intense liée à la villégiature a ćté le précurseur de la notoriété et de la crédibilité de plusieurs régions touristiques du Québec. C'est cette présence qui a suscité la venue et le développement de stations où s'ajoutent maintenant hottels, condominiums, apart-hôtels, auberges, centre de villégiature en plus d'une gamme diversifiée de services et d'activités récréo-touristiques et culturelles. Pourtant, on se demande encore dans certains milieux si le tourisme de villégiature fait vraiment bien partie de l'industrie touristique, si c'est du vrai tourisme. Ainsi, on reconnailt par exemple que Sutton est une station touristique hivernale à cause de son centre de ski alpin alors qu'on l'oublie presque complètement du décor touristique estrien durant la saison estivale méme si on peut compter 550 a 600 résidences secondaires dans le secteur. Bien plus; dans le devis préparé par le ministère du Tourisme du

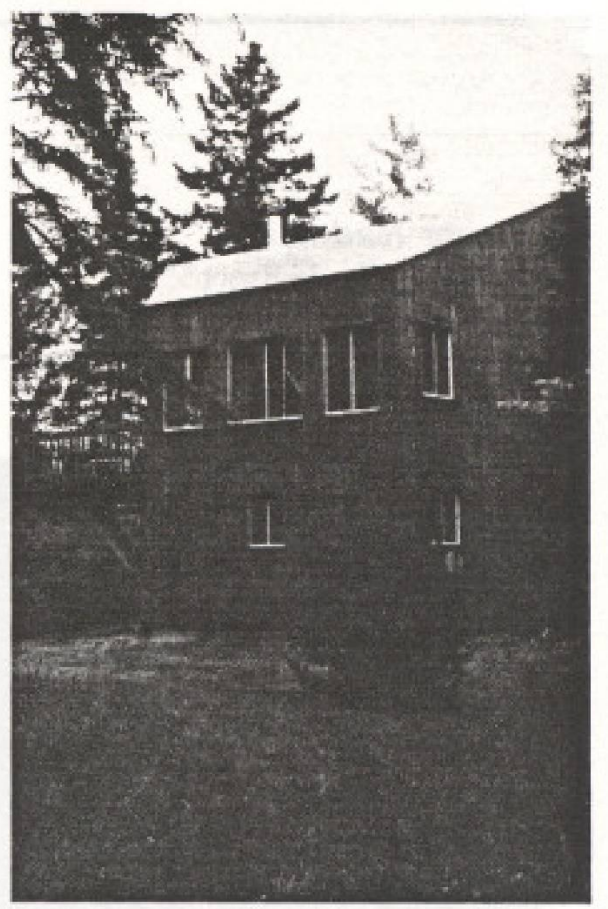

Québec dans le cadre des Plans de développement touristique de chacune des ATR du Québec, le tourisme de villégiature a été complètement évacué, mis de côté; ce qui nous apparaît être une aberration totale. Comment peut-on penser décrire la problématique touristique d'une région comme l'Estrie et procéder à une évaluation globale de l'offre et de la demande sans tenir compte de ses 18500 chalets et de son demi-milliard de dollars en valeur fonciere?? "A cause du facteur répétitif, à cause du séjour trop long, parce que ce sont des Québécois qui dépensent au Québec" nous a-t-on répondu. Doiton en conclure que les ménages québécois qui en seront cette année à leur $19 \mathrm{e}$ vacance annuelle sur les plages du Maine ne doivent plus être considérés comme des touristes? Doit-on en conclure que les milliers de ménages québécois qui vont passer l'hiver en Floride devraient être exclus des statistiques touristiques? Doit-on en conclure que les milliards de dollars dépensés au Québec par les villégiateurs sont moins importants que les centaines de millions de dollars qui nous sont apportés par les étrangers en visite chez nous? Oui, vraiment, le tourisme de villégiature est le mal aimé de notre industrie touristique!

\section{La villégiature en Estrie}

Le tableau 1 donne une idée assez précise de l'état de la villégiature en résidences secondaires en Estrie, ainsi que son évolution numérique au cours des quatre dernières années. On observe que dans la plupart des MRC de la région, le nombre de résidences secondaires connaît une légère baisse, ce qui se répercute bien sûr sur la population saisonnierre. Le nombre de chalets est passé de 18408 à 17840 entre 1984 et 1987 , alors que pendant la même période, la population saisonnière passait de 88378 à 70726 . Par 
TABLEAU 1

Nombre de résidences secondaires, population saisonnière* et

tvaluation foncière, 1984 et 1987, région de l'Estrie, par MRC

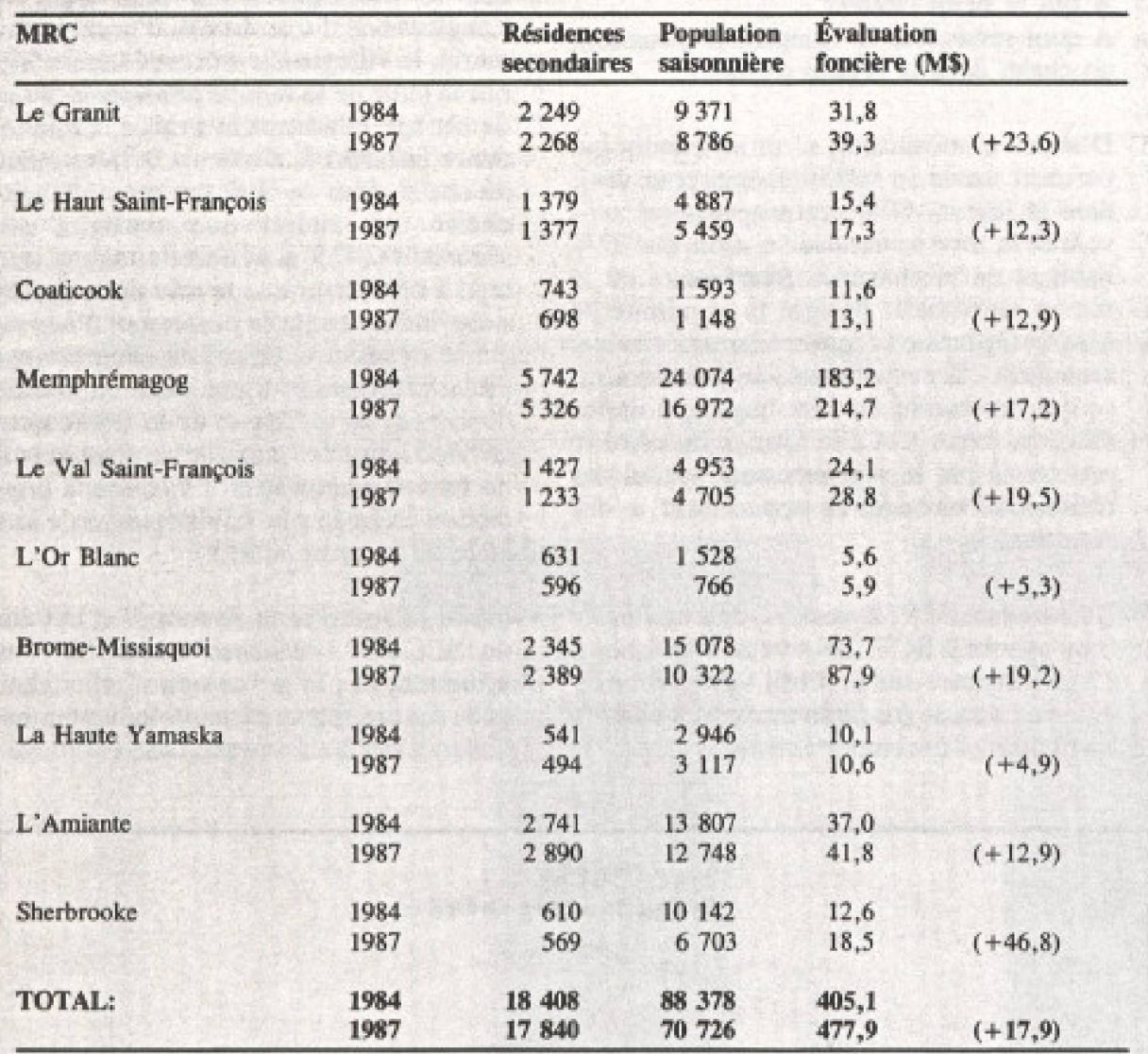

Source: Ministetre des Affaires municipales, 1984 et 1987.

*: Estimations fournies par chacune des municipalités.

Tableau: Roger Nadeau, Université de Sherbrooke.

rôles d'évaluation des quatorze municipalités de la zone, alors que les données plus approfondies ont été ramassées à l'aide d'un questionnaire envoyé à un échantillon représentatif de propriétaires de chalets. Cet échantillon sélectionné scientifiquement tient également compte d'une représentativité par municipalité. Sur un total de 464 questionnaires, 193 ont été retournés et acceptés, apportant un taux de réussite parfaitement valable statistiquement $(41,6 \%)^{(14)}$. L'organisation mondiale du tourisme affirme qu'un taux de $40 \%$ a $50 \%$ est rarement obtenu dans ce genre d'enquête ${ }^{(15)}$.

\section{Un poids lourd ou léger?}

La région de Magog-Orford comptait 4644 résidences secondaires en 1984 , soit $35 \%$ du stock résidentiel (figure 1). Le taux de fonction résidentielle (TFR) tel que proposé par Barbier ${ }^{(16)}$ atteint pour la région $54 \%$, taux qui approche dangereusement le seuil de dépendance. Lorsqu'une région détient un TFR de plus de $60 \%$, on peut affirmer que le phénomène est une ressource essentielle au développement de cette zone.

Monétairement, la villégiature privée allait chercher $22,5 \%$ de la valeur d'évaluation totale régionale, soit plus de $146000000 \$$ (de 1984). Il existe cependant des écarts locaux qui permettent d'identifier des secteurs ou localités particulièrement vulnérables face à la villégiature, alors que plus de $50 \%$ du budget provenait des taxes payées par les villégiateurs. La valeur moyenne d'une résidence secondaire dans MagogOrford était de $32300 \$$ avec des écarts moyens locaux yariant de 175005 à 53000 s.

La villégiature privée apparaît comme un secteur d'activité particulièrement important pour la région; les immobilisations à elles seules contribuent largement à la santé administrative de certaines localités.

contre, l'évaluation foncière connaissait un bond de $17,9 \%$, passant de $\$ 405,1$ millions à $\$ 477,9$ millions. Nous sommes donc tout près du demi-milliard de dollars: ça commence à valoir la peine de prendre le phénomène au sérieux! En Estrie, l'évaluation foncière moyenne se situe à 26788 , une des plus élevée au Québec.

Une brève analyse permet de constater que d'une MRC à l'autre, les disparités peuvent être fort importantes; on verra au chapitre suivant, qu'à l'intérieur même d'une MRC, les Ecarts peuvent être très prononés d'une municipalité à l'autre. Ainsi, si l'évaluation foncière moyenne se situe à 26788 \$, Memphrémagog avec $40368 \$$, BromeMissisquoi avec $36835 \$$ et Sherbrooke avec $32513 \$$ sont largement au-dessus de la moyenne alors que le Haut St-François et l'Or Blanc ferment la marche avec des moyennes respectives de $12563 \$$ et $9900 \$$. Par ailleurs, on observe des écarts tout aussi importants en ce qui a trait au nombre moyen de villégiateurs qui séjournent à la résidence secondaire. Selon les données recueillies par le ministère des Affaires municipales, cette moyenne se situerait à 3,9 en Estrie, mais elle atteindrait 11,6 dans la MRC de Sherbrooke et 6,3 dans Brome-Missisquoi, comparativement à 1,6 dans la MRC de Coaticook et 1,3 dans l'Or Blanc.

\section{Magog-Orford à la loupe}

Le portrait d'ensemble dressé dans les prochaines lignes a été tiré d'une étude produite en 1984 sur la villégiature privée dans la région de Magog-Orford en Estrie ${ }^{(12)}$. En plus de vérifier les implications sociales, spatiales, économiques et environnementales de la villégiature sur le territoire, cette recherche a porté sur l'évolution de cette forme de tourisme, par la comparaison avec une autre étude réalisếe en 1971 sur le mềme secteur ${ }^{(13)}$.

Le choix de la région de Magog-Orford a découlé, bien sûr de cette possibilité de comparer, mais aussi de l'intérêt que suscite cette zone pour son potentiel touristique et de $1^{+}$implantation historique du phénomène sur ses terres. L'obtention des données de base provient de deux sources: les données d'ensemble ont êté̉ recueillie à même les
Comparativement aux données de Nadeau en $1968^{117}$, notre étude apporte la vérification d'un comportement relativement récent des villégiateurs. Sur les douze municipalités étudiées dans les deux études (figure 1), seulement 309 résidences secondaires s'y seraient ajoutées. Cette faible progression est d'autant moins réaliste que dans le seul canton d'Orford, plus de 300 chalets ont été construits depuis 1968. On peut donc croire que l'excédent de certaines localités a été annihilé par la perte quantitative d'autres municipalités. Ce déficit serait attribuable à la mutation résidentielle, pratique qui consiste à transformer le chalet en domicile permanent. Ce comportement s'est davantage produit dans les secteurs à proximité de Sherbrooke, ville hébergeant un grand nombre de villégiateurs de la région. L'origine du propriétaire liée au facteur distance semble être une des motivations majeures de transformation $d u$ chalet en domicile permanent.

Bien qu'en 1984, le TFR s'approchait du seuil de dépendance, il faut mentionner que 
ce taux a régressé depuis 1968 de plus de la moitié. Autre diminution notable, le pourcentage de l'évaluation foncière provenant des résidences secondaires: cette forme de résidence comptait pour $50 \%$ de la valeur régionale en 1968 par rapport à $20 \%$ en 1984.

Le poids de la villégiature privée dans la région Magog-Orford, bien qu'encore important, a connu une baisse résultant de l'activité d'autres types d'immobilisations, qui permet de décentraliser progressivement le pouvoir qu'exerçait cette forme de tourisme sur l'action ou l'inertie de certaines autorités locales.

\section{La haut sur la montagne...}

C'est avant tout grâce à sa montagne et aux nombreux lacs qu'elle abrite que la région a su attirer ses quelque 4600 propriétaires de chalet. La villégiature privée est depuis longtemps associée à l'élément lacustre. Voilà pourquoi on a envahi les extrémités des lacs pour ensuite s'étendre sur les rives adjacentes de qualité. On a même défié̉ des rivages inaptes à la construction dans le but ultime d'avoir un accès direct avec l'eau. Apparurent finalement les développements linéaires double, triple et même des développements densément lotis. Cet acharnement sur l'élément lacustre de la région Magog-Orford apporte comme conséquence une saturation (voire une sursaturation dans certains cas) du couloir riverain régional. Certes, certains lacs demeurent encore accessibles, mais la majorité se retrouve avec un déséquilibre de leur environnement inhérent à quatre actions de la villégiature privée en particulier: artificialisation, déboisement, densification et surutilisation du couloir lacustre.

En analysant l'étendue spatiale du phénomène depuis 1971 et à la lumière des résultats de notre enquête, il appert que les propriétaires de chalet ont constaté cette saturation et ont réagi en s'adaptant à un nouveau milieu d'implantation, le milieu non-riverain (plus de 300 mètres d'un lac). Concept non-observé en 1971, ce milieu accueillait en 1984, 19\% des résidences secondaires de la région (environ 880). Cette transition du lac à la montagne origine de cinq motivations principales du propriétaire:

- qualité du paysage non-riverain (panorama et nature)

- tranquilité du secteur montagneux

- prix d'achat plus raisonnable en milieu non-riverain

- rareté et surenchère des terrains lacustres

- la pratique de sports d'hiver lice à la montagne

On ne peut prétendre que cette transition s'applique à toutes les régions du Québec puisque son apparition a été conditionnée par deux éléments: la saturation du couloir riverain et la présence d'un relief attrayant. plus un facteur conditionnel à l'implantation d'une résidence secondaire dans le secteur Magog-Orford.

\section{À qui le beau chalet?}

À quoi ressemble le villégiateur possédant un chalet dans la région d'étude?

D'abord et avant tout, c'est un citadin qui parcourt moins de 160 kilomètres pour venir dans la région. $50 \%$ des propriétaires arrivent de la zone montréalaise, alors que $32 \%$ habitent en permanence Sherbrooke ou la région immédiate. Malgré la proximité de Magog-Orford avec la frontière américaine, seulement $2 \%$ des villégiateurs la traversent, ce qui représente un taux beaucoup moins élevé qu'anticipé. Cette illusion du nombre est causée par le regroupement spatial des résidences secondaires appartenant à des Américains.

Québécois dans $97 \%$ des cas, il est toutefois francophone à $84 \%$. Le groupe linguistique a une influence sur le profil villégiateur de la région à cause des différences existant dans les mentalités et comportements. Le villégia- teur a en moyenne 51 ans alors que la moyenne québécoise pour les 25 ans et plus était de 46 ans en $1981^{(18)}$. L'âge du propriétaire de chalet varie selon la langue, puisque le francophone est plus jeune que l'anglophone d'une dizaine d'années. Étant marié, le villégiateur voit cependant s'affaiblir la taille de sa famille dépendante à cause de cet âge relativement avancé. Cette incidence influencera d'ailleurs la fréquentation du chalet. Plus de $60 \%$ des propriétaires de chalet ont atteint une scolarité postsecondaire ( $42 \%$ sont universitaires), ce qui tend à démontrer que le niveau de scolarité a une influence sur la possession d'une résidence secondaire. Cette constatation contredit les affirmations d'une étude du ministère du Loisir, de la Chasse et de la Pêche qui, en 1983, disait: "tla scolarité ne semble pas un facteur déterminant ${ }^{+(19)}$. Quant à la profession exercée par le villégiateur, elle est libérale ou cadre à $60 \%(20)$.

Même si Jean-Pierre St-Amour ${ }^{(21)}$ et l'étude du M.L.C.P. affirmaient que le revenu $\mathrm{n}$ 'influençait pas la possession d'un chalet, nous devons rejeter cette position pour notre région d'étude. Le revenu médian des ména-

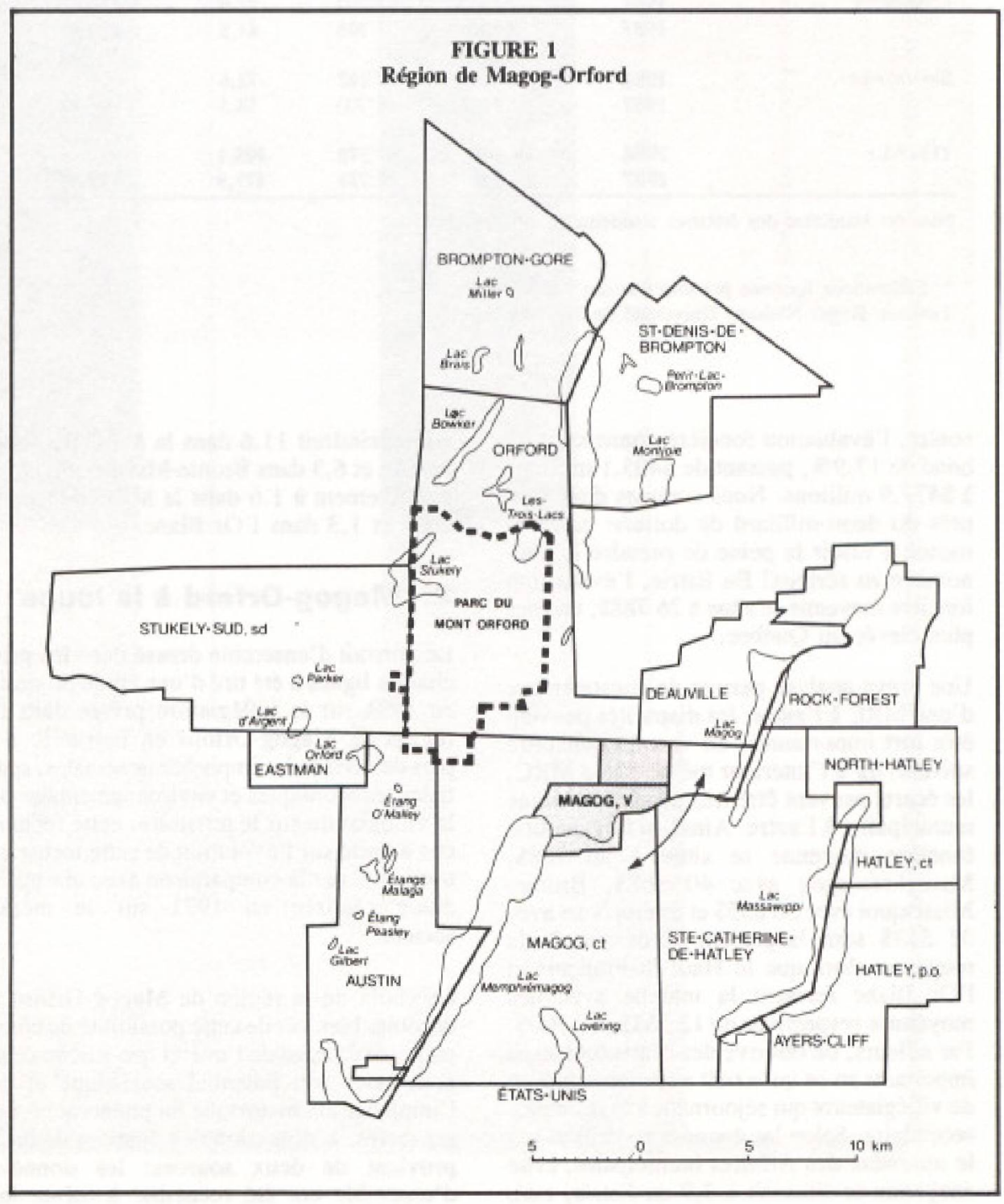




TABLEAU 2
Evolution des jours de villégiature privée dans Magog-Orford
pour les 12 municipalittés comparés

ges villégiateurs était de $45000 \$$ en 1984 comparativement à $20000 \$$ en 1981 pour tous les ménages du Québec. $50 \%$ des ménages propriétaires d'un chalet dans MagogOrford ont un revenu de $40000 \$$ ou plus, par rapport à $12,5 \%$ pour les ménages de l'ensemble québécois.

Il existe une série de relations entre les variables socio-professionnelles difficilement dissociables, laissant entrevoir un enchaînement de cause à effet qui conduira ou non à la possession d'un chalet.

On peut affirmer que même si la villégiature privée n'est pas exclusive à une classe sociale précise, comme le disait Saint-Amour, ce mode d'hébergement favorise surtout les mieux nantis, du moins en ce qui concerne la région de Magog-Orford.

La comparaison du profil entre 1971 et 1984 démontre qu'il subit les effets du temps de la même manière que celui de la population québécoise: vieillissement, taux de divorcés accru, famille moins nombreuse, études plus avancées. Par contre, ce profil s'est modifié en accentuant certaines tendances discriminantes face à la possession d'un chalet: scolarité élevée, profession de classe supérieure, revenu toujours plus grand.

$\mathrm{La}$ langue et le revenu sont deux variables dont les écarts depuis 1971 sont très importants. En 13 années, le taux d'anglophones villégiateurs a chuté de $38 \%$ à $14,7 \%$. Quant au revenu, en ramenant le dollar de 1971 à celui de 1984, on accorde au propriétaire de chalet de la région un accroissement de son revenu médian de $12600 \$$ en 13 ans. Cette augmentation provient partiellement du travail des deux conjoints, pratique moins populaire au début des années 1970.

\section{Un produit sous-utilisé}

De par son nom la résidence secondaire ne peut être utilisée en permanence puisque pour demeurer secondaire, le propriétaire doit se restreindre à un maximum de 182 jours par année d'utilisation. Cependant, le maximum est loin d'étre atteint dans la région puisque la médiane du séjour d'un propriétaire de chalet atteint 58 jours/année. Ces journées de séjour sont inégalement réparties, $65 \%$ des résidences secondaires de la zone étant exclusivement utilisées en été. On préfere les fins de semaine et les vacances pour venir au chalet.
Puisque la taille médiane d'un ménage propriétaire séjournant au chalet est de 3 personnes, chaque résidence secondaire voit son taux d'occupation passer à 174 jours par an ( $3 \times 58$ jours), ce qui apporte à l'ensemble régional 809100 jours/personne de villégiateur privee ( 174 jours $\times 4650$ chalets).

En ajoutant à cette clientèle régulière une clientèle occasionnelle qui regroupe les invités venant au chalet, on doit ajouter 139500 jours/personne de villégiature privée $\mathrm{e}^{(22)}$ à la région pour un total pondérable de 948600 journées. Pondérable, parce qu'il faut penser à la clientèle temporaire qui regroupe les locataires d'un chalet pour une ou deux semaines et même davantage ${ }^{(23)}$.

Par rapport à 1971, le villégiateur a grandement modifié la durée et la fréquence d'utilisation du chalet. Une plus grande fréquentation les fins de semaine liée à une baisse de popularité du séjour pour tout un été ont contribué à réduire l'importance du séjour estival (83 jours en 1971 à 53 jours en 1984). Inversement, le séjour hivernal se popularise alors que $63 \%$ des propriétaires vont au chalet durant cette saison comparativement à $41 \%$ en 1971. Annuellement, la durée du séjour est passée de 90 à 58 jours en 13 années. Ce facteur, jumelé à une diminution de la taille du ménage propriétaire et à un nombre d'invités moins important, a conduit à une perte de 798194 journées/personne de villégiature privée dans la région d'étude.

A cause de l'évolution constatée depuis 1971. on peut affirmer que la résidence secondaire dans Magog-Orford est nettement sousutilisée par rapport à son taux d'occupation potentiel, d'où la qualification "bien de luxe" qu'on lui attribue.

\section{Apport économique + résidence} secondaire $=$ réalité rentable

Discuter du pouvoir économique de la villégiature sur un territoire d'accueil demeure un sujet délicat et difficilement pondérable. Coppock $^{(24)}$ et Cribier ${ }^{(25)}$ ont soulevé les contradictions qui existaient dans la définition de l'importance de cet impact. Cependant, malgré la réticence de certains auteurs, il apparaît hors de tout doute que la villégiature privée influence l'économie de la région. Notre étude a pesé sur cette influence en tenant compte, et de la dépense totale qu'apporte un chalet, et de la proportion de cette dépense effectuée dans la région où se localise la résidence secondaire. En 1984, l'enquête a permis d'évaluer à $3900 \$$ la dépense moyenne annuelle directement attribuable à la possession d'un chalet, ce qui représente plus de 175000005 pour l'ensemble de la villégiature privée localisée dans Magog-Orford. Par rapport à d'autres études réalisées ces dernières années, la dépense calculée dans notre sondage est très réaliste. Le Muskoka Lake Association (1984) en Ontario ${ }^{(26)}$ a établi à $4700 \$$ la dépense totale moyenne pour un chalet dans cette région et la FAPEL (1982) a evalué a $3838 \$$ cette dépense pour l'ensemble du Québec ${ }^{[27}$. Finalement, le MLCP (1983) avec un chiffre estime a $1243 \$$ avec erreur-standard de $95,10 \$$ pour l'ensemble québécois va à $l^{*}$ encontre de tous les autres résultats ${ }^{(28)}$.

Cependant, cette dépense totale subit de nombreuses variations en fonction du profil du propriétaire et du territoire étudié, de sorte qu'il est difficile d'apporter une évaluation parfaitement conforme à la réalité. On a cependant constaté dans la région MagogOrford que la dépense totale était en relation étroite avec le revenu du ménage, la dimension du chalet, la valeur de ce dernier et la durée du séjour. En outre, il semblerait que la valeur foncière de la résidence secondaire précisée au rôle d'évaluation permettrait de calculer assez précisément ( $102 \%$ dans notre cas) la dépense totale effectuée par les propriétaires de chalet. Nous avons pu évaluer l'apport réel de la villégiature privée dans l'économie régionale. Même si une partie des dépenses encourues ne représentent qu'une transposition du domicile permanent au chalet (exemple, la nourriture), d'autres dépenses constituent un surplus que doit supporter le villégiateur.

Chaque propriétaire effectuant $72 \%$ de ses dépenses dans la région de localisation du chalet, soit un montant moyen de 2798 \$ par an, la villégiature privée injecte dans l'économie régionale plus de $12500000 \$$ annuellement.

On notera que ce chiffre ne tient pas compte de l'impact des clientèles occasionnelles et temporaires qui bénéficient également de ce mode d'hébergement et qui contribuent sûrement à l'économie de la zone. Une analyse détaillée de la somme dépensée en région en tenant compte des variables socioprofessionnelles du propriétaire de chalet nous permet d'affirmer que la distance entre le domicile permanent et la résidence secondaire est déterminante. En effet, plus le propriétaire est éloigné de son chalet, plus ses dépenses seront effectuées dans le secteur entourant sa résidence secondaire, et plus le montant de ses dépenses sera élevé.

Le point de chute de ces dépenses (en excluant les taxes foncières) profite tout particulièrement aux centres urbains de Magog et Sherbrooke, avec $6481000 \$$ et $1342000 \$$ respectivement. Les centres secondaires tirent davantage profit de cet impact au niveau des dépenses courantes et pour le loisir. 
TABLEAU 3

\begin{tabular}{lccc}
\hline Origine & $\begin{array}{c}\text { Dépense en } \\
\text { région }\end{array}$ & $\begin{array}{c}\text { Dépense } \\
\text { totale }\end{array}$ & $\begin{array}{c}\text { \% dépensé } \\
\text { en rćgion }\end{array}$ \\
\hline Montréal (ile) & 3775 & 4690 & 80,5 \\
Montréal (banlieue) & 2922 & 3785 & 77,2 \\
Rive Sud (ouest) & 2296 & 3375 & 68,0 \\
Sherbrooke & 1696 & 3454 & 49,1 \\
Région immédiate & 1159 & 2095 & 55,3 \\
\hline
\end{tabular}

En considération de l'évolution de cet impact économique depuis 1971, il appert que la contribution de la villégiature privée dans l'activité économique de la région MagogOrford a considérablement régressé. Concrètement, c'est $1000 \$$ (actualisé en dollars de 1984) de moins que chaque propriétaire dépense annuellement dans la zone. La région aurait perdu plus de 35000005 émanant de cette forme de tourisme depuis 13 ans. Il faut cependant considérer que c'est la dépense totale annuelle qui a diminué, ce qui a logiquement conduit à une baisse de la dépense régionale. Les causes de diminution de la dépense inhérente à la possession et l'utilisation d'un chalet seraient associables à 6 facteurs explicatifs:

- proportion moindre d'anglophones, groupe qui dépensait davantage

- temps de séjour au chalet réduit

- taille du ménage propriétaire plus petite

- moins d'invités venant au chalet

- des chalets plus petits en dimension

- qualité des services et du réseau d'accès à ces services mis en doute.

Malgré tout, la villégiature privée demeure un secteur important de l'activité économique régionale qui, avec un impact environnemental négatif, ne fait que confirmer l'éternelle problématique qui entoure ce phénomène: doit-on ou non encourager le développement de cette forme de tourisme? Nous sommes tentés de répondre dans l'affirmative à la condition d'appliquer de façon stricte deux principes fondamentaux: planification et réglementation.

\section{Peut-on extrapoler?}

Après s sêtre adonné à une étude assez poussée de l'impact de la villégiature dans une région, on est normalement porté à se demander quel serait cet impact à l'échelle du Québec. Voilà une question intéressante pour qui aime vivre dangeureusement!

Prétendre que les 18000 résidences secondaires de l'Estrie sont représentatives des 300000 que compte le Québec serait sans doute plutôt risqué. Nous avons donc cherché des données supplémentaires susceptibles de renforcir la validité d'une éventuelle extrapolation et nous avons réussi à mettre la main sur le fichier du ministère des Affaires municipales qui conserve en mémoire toutes les informations nécessaires à la confection des röles d'évaluation, dont celles portant sur les résidences secondaires, pour chacune des municipalités du Québec. Nous avons regroupé les municipalités par Municipalité Régionale de Comté, et les MRC par Association Touristique Régionale. Ces compilations nous ont permis d'obtenir le portrait des $96 \mathrm{MRC}$ (plus deux Communautés Urbaines), regroupées en 16 ATR (celles de Montréal et du Nouveau-Québec n'étant pas incluses) (voir tableau 4). Après avoir pesé la touche "total" sur la calculatrice, on obtient 199041 résidences secondaires au Québec en 1987. Ce "ttotal" n'est pas sans causer un choc, surtout parce qu'en 1981, l'étude du MLCP révélait que 302300 ménages québécois possédaient une résidence secondaire; normalement, en 1987, on devrait s'attendre à en trouver 325 ou $340000 \ldots$ mais le ministère des Affaires municipales n'en recense plus qu'un peu moins de 200000 . Bien qu'habitué à la précarité des données touristiques, ce trou a de quoi laisser perplexe car, enfin, 100000 chalets, ça ne disparait pas comme ça!

En retournant aux sources, nous apprenons que les relevés du MAM "exclus tous les logements qui ne sont pas fixés au sol, non sujet à taxation (...). De plus, il y a sans doute un nombre additionnel considérable de résidences qui sont bâties sur des territoires non organisés...". Quant aux relevés du MLCP, ils "incluent tous les genres d'hébergement privés de loisir, y compris les roulottes de camping et les maisons mobiles. L'estimation compte toutes les résidences de villégiature des territoires municipalisés et des territoires non organisês."

Notre définition est assez conforme à celle du ministère des Affaires municipales mais son relevé est incomplet à cause d'un "nombre additionnel considérable de résidences qui sont băties sur des territoires non organisés". Le nombre réel de résidences secondaires au Québec, dans le sens des définitions retenues pour le présent document, est donc supérieur à 200000 mais inférieur à 300000 . Il serait étonnant que ce nombre dépasse 220000 unités. S'il est raisonnable de ten- ter une extrapolation à partir de cette donnée, l'évaluation foncière des résidences. secondaires privées au Québec atteindrait approximativement $4350000000 \$$ en 1987 , soit en moyenne de près de $20000 \$$ par propriété. En outre, pas moins de un million de personnes auraient séjourné au chalet en 1986; comme ce séjour moyen s'étend sur environ 60 jours, on peut alors dire qu'il s'est passé 60000000 jours/personnes de villégiature en chalets privés au Québec l'année dernière.

Tentons une dernière extrapolation portant sur l'impact économique découlant de l'utilisation de 220000 résidences secondaires. L'étude sur Magog-Orford évalue à $3900 \$$ la dépense moyenne annuelle directement attribuable a la possession d'un chalet mais encore ici, il est plus que probable que le secteur de Magog-Orford ne soit pas représentatif de l'ensemble des stocks québécois de résidences secondaires. En effet, l'évaluation foncière moyenne par chalet au Québec s'établit à 20000 \$; cette moyenne atteint 26800 \$ pour l'ATR de l'Estrie et dépasse le $40000 \$$ dans la MRC de Memphrémagog. Par contre, l'étude de FAPEL qui porte sur l'ensemble du Québec obtenait la même dépense moyenne annuelle que nous, alors que l'étude ontarienne citée précédemment atteignait même 4700 \$. Si nous prenions donc pour acquis que cette somme de $3900 \$$ pourrait bien être représentative, cela signifierait que les villégiateurs québécois dépensent annuellement un peu plus de $850000000 \$$ pour pouvoir séjourner à leur chalet, et que de cette somme, 615000000 s sont dépensés en région... annuellement toujours!

\section{La villégiature en copropriété}

Sous le titre de "résidences secondaires", l'hebdomadaire Les Affaires publiait au début d'avril 1988 une image de la villégiature en Estrie mais sous l'angle "'condominium ". II ne s'agit donc pas du type d'hébergement dont il est question dans les quatre premières parties de ce document mais pour avoir une vision complète de l'intérêt de la villégiature chez nous, il est sans doute utile de voir comment s'y comporte ce type d'établissement. Comme le journal Les Affaires vient de faire son enquête, nous nous inspirerons grandement de ses propos ${ }^{(30)}$.

"Il y a très peu de résidences secondaires à vendre dans la région de Magog-Orford. Et lorsqu'ils en trowvent une, les acheteurs doivent s'attendre a payer plus de 100000 s. Par contre, les projets de nouveaux condominiums croissent en nombre, et très vite! Dans les seuls cantons de Magog et d'Orford, on construira pas moins de 300 condominiums cette année. Au Lac Brome, whe centaine de résidences secondaires s'ajouteront aux 250 qui ont été érigées l'an dernier".

Pour M. André Felton, agent immobilier: "pour l'instant, le lac Memphrémagog reste 
TABLEAU 4

Nombre de résidences secondaires, population saisonnière*, évaluation foncière, au Québec, 1984 et 1987, par ATR

\begin{tabular}{|c|c|c|c|c|c|c|}
\hline$\overline{A T R}$ & & $\begin{array}{l}\text { Résidences } \\
\text { secondaires }\end{array}$ & $\begin{array}{l}\text { Estimation } \\
\text { s population } \\
\text { saisonnietere }\end{array}$ & $\begin{array}{l}\text { Evaluati } \\
\text { foncière }\end{array}$ & (M\$) & $\begin{array}{l}\text { Valeur } \\
\text { moyenne par } \\
\text { chalet (1987) }\end{array}$ \\
\hline $\begin{array}{l}\text { Iles-de-la-Madeleine } \\
\text { (1 MRC) }\end{array}$ & $\begin{array}{l}1984 \\
1987\end{array}$ & $\begin{array}{l}417 \\
331\end{array}$ & $\begin{array}{l}9272 \\
8758\end{array}$ & $\begin{array}{l}4,0 \\
4,7\end{array}$ & $+14,8 \%$ & 14199 \\
\hline $\begin{array}{l}\text { Gaspésie } \\
\text { (8 MRC) }\end{array}$ & $\begin{array}{l}1984 \\
1987\end{array}$ & $\begin{array}{l}5872 \\
6023\end{array}$ & $\begin{array}{l}52011 \\
48660\end{array}$ & $\begin{array}{l}57,6 \\
65,6\end{array}$ & $+13,8 \%$ & 10891 \\
\hline $\begin{array}{l}\text { Bas St-Laurent } \\
\text { (5 MRC) }\end{array}$ & $\begin{array}{l}1984 \\
1987\end{array}$ & $\begin{array}{l}6600 \\
6662\end{array}$ & $\begin{array}{l}19779 \\
22428\end{array}$ & $\begin{array}{l}73,2 \\
82,2\end{array}$ & $+10,9 \%$ & 12188 \\
\hline $\begin{array}{l}\text { Québec } \\
(4+1 \mathrm{MRC})\end{array}$ & $\begin{array}{l}1984 \\
1987\end{array}$ & $\begin{array}{l}8723 \\
8483\end{array}$ & $\begin{array}{l}40140 \\
35940\end{array}$ & $\begin{array}{l}167,3 \\
186,7\end{array}$ & $+11,6 \%$ & 22009 \\
\hline $\begin{array}{l}\text { Charlevoix } \\
\text { (2 MRC) }\end{array}$ & $\begin{array}{l}1984 \\
1987\end{array}$ & $\begin{array}{l}2051 \\
2082\end{array}$ & $\begin{array}{l}16097 \\
15952\end{array}$ & $\begin{array}{l}33,0 \\
38,7\end{array}$ & $+17,2 \%$ & 18528 \\
\hline $\begin{array}{l}\text { Pays de l'Érable } \\
\text { (10 MRC) }\end{array}$ & $\begin{array}{l}1984 \\
1987\end{array}$ & $\begin{array}{l}12478 \\
12070\end{array}$ & $\begin{array}{l}38260 \\
35033\end{array}$ & $\begin{array}{l}147,0 \\
184,0\end{array}$ & $+25,1 \%$ & 15244 \\
\hline $\begin{array}{l}\text { Cocur du Québec } \\
\text { (10 MRC) }\end{array}$ & $\begin{array}{l}1984 \\
1987\end{array}$ & $\begin{array}{l}17020 \\
19345\end{array}$ & $\begin{array}{l}71326 \\
72553\end{array}$ & $\begin{array}{l}190,4 \\
272,3\end{array}$ & $+43,0$ & 14076 \\
\hline $\begin{array}{l}\text { Estrie } \\
\text { (10 MRC) }\end{array}$ & $\begin{array}{l}1984 \\
1987\end{array}$ & $\begin{array}{l}18408 \\
17840\end{array}$ & $\begin{array}{l}88378 \\
70726\end{array}$ & $\begin{array}{l}405,1 \\
477,9\end{array}$ & $+17,9 \%$ & 26788 \\
\hline $\begin{array}{l}\text { Laurentides } \\
\text { (8 MRC) }\end{array}$ & $\begin{array}{l}1984 \\
1987\end{array}$ & $\begin{array}{l}42442 \\
39320\end{array}$ & $\begin{array}{l}239204 \\
232614\end{array}$ & $\begin{array}{r}948,8 \\
1026,6\end{array}$ & $+8,2 \%$ & 26109 \\
\hline $\begin{array}{l}\text { Outaouais } \\
\text { (4 MRC) }\end{array}$ & $\begin{array}{l}1984 \\
1987\end{array}$ & $\begin{array}{l}14150 \\
19217\end{array}$ & $\begin{array}{l}60643 \\
80338\end{array}$ & $\begin{array}{l}453,9 \\
518,1\end{array}$ & $+14,1 \%$ & 26960 \\
\hline $\begin{array}{l}\text { Montérégie } \\
\text { (13 MRC) }\end{array}$ & $\begin{array}{l}1984 \\
1987\end{array}$ & $\begin{array}{l}13418 \\
12549\end{array}$ & $\begin{array}{ll}74 & 126 \\
72 & 129\end{array}$ & $\begin{array}{l}231,1 \\
251,7\end{array}$ & $+8,9 \%$ & 20057 \\
\hline $\begin{array}{l}\text { Lanaudière } \\
\text { (6 MRC) }\end{array}$ & $\begin{array}{l}1984 \\
1987\end{array}$ & $\begin{array}{l}27469 \\
26204\end{array}$ & $\begin{array}{l}183414 \\
143358\end{array}$ & $\begin{array}{l}459,4 \\
534,8\end{array}$ & $+16,4 \%$ & 20409 \\
\hline $\begin{array}{l}\text { Abitibi-Témiscamingue } \\
\text { ( } 5 \mathrm{MRC} \text { ) }\end{array}$ & $\begin{array}{l}1984 \\
1987\end{array}$ & $\begin{array}{r}10009 \\
8878\end{array}$ & $\begin{array}{l}30412 \\
26067\end{array}$ & $\begin{array}{l}67,3 \\
81,5\end{array}$ & $+20,8 \%$ & 9180 \\
\hline $\begin{array}{l}\text { Saguenay - Lac St-Jean - } \\
\text { Chibougamau } \\
\text { (4 MRC) }\end{array}$ & $\begin{array}{l}1984 \\
1987\end{array}$ & $\begin{array}{l}12036 \\
15946\end{array}$ & $\begin{array}{l}53403 \\
54930\end{array}$ & $\begin{array}{l}126,3 \\
168,3\end{array}$ & $+33,2 \%$ & 10554 \\
\hline $\begin{array}{l}\text { Manicouagan } \\
(2 \mathrm{MRC})\end{array}$ & $\begin{array}{l}1984 \\
1987\end{array}$ & $\begin{array}{l}2571 \\
2716\end{array}$ & $\begin{array}{l}5109 \\
5418\end{array}$ & $\begin{array}{l}18,0 \\
20,8\end{array}$ & $+15,5 \%$ & 7658 \\
\hline $\begin{array}{l}\text { Duplessis } \\
\text { (3 MRC) }\end{array}$ & $\begin{array}{l}1984 \\
1987\end{array}$ & $\begin{array}{l}1316 \\
1375\end{array}$ & $\begin{array}{ll}4 & 120 \\
2733\end{array}$ & $\begin{array}{l}11,2 \\
12,2\end{array}$ & $+8,9 \%$ & 8873 \\
\hline $\begin{array}{l}16 \text { ATR } \\
\text { ou } \\
96 \mathrm{MRC}\end{array}$ & 1984 & 194980 & $\begin{array}{r}1075694 \\
(5.5) \\
927637 \\
(4.6)\end{array}$ & 3393,6 & $+15,6 \%$ & 19720 \\
\hline
\end{tabular}

Source: Ministère des Affaires municipales; méthode uniformisée d'évaluation foncière.

Collaboration: Jacques Luneau, Analyse et développement, ministère du Tourisme du Québec, avril 1988.

* Estimations fournies par chacune des municipalités.

Tableau: Roger Nadeau, Université de Sherbrooke. touristique du Mont Orford prévoit construire 125 nouvelles unités à son projet du village Mont Orford au cours des 18 prochains mois. Aux Villas de l'Anse, dans le canton de Magog, sur la rive est du lac Memphrémagog, on a vendu 110 terrains et construit 60 villas au cours des deux dernières années.

"Dans le canton d'Orford, les projets de l'Auberge Estrimont et le Jardin des Sables continuent leur expansion en lançant de nouvelles phases de construction. Le prix des villas vont d'un peu moins de 100000 \$ jusqu'à $200000 \$$. La majorité des acheteurs destinent leur propriété à la location. Les deux complexes comptent déjà chacun une centaine d'unites"s".

À Bromont, Robert Désourdy "vient de lancer Le Bromont Club, un projet de maisons en rangée se vendant entre $120000 \$$ et 178000 \$. Ce projet est situé sur le Mont Soleil entre deux pistes de ski. On y accédera en voiture en passant dans un tunnel sous une pente de ski". Le projet compte 56 unités.

Situé sur la rive ouest du Lac Brome, le Manoir Inverness comptera 120 unités au mois de juillet. Il en reste actuellement une vingtaine à vendre, à un prix variant entre $100000 \$$ et $150000 \$$. Cent soixante-quinze des 300 acres du projet ont été retenu pour aménager un golf de 18 trous. Le terrain inutilisé permet une expansion future puisque les bắtisses existantes $n^{*}$ occupent que 24 acres. De l'autre côté du lac, sur la rive est, l'Auberge du Lac Brome a déjà vendu 102 unités de copropriété en un an, entre 80000 \$ et $190000 \$$. Un peu plus loin, l'Auberge Le Viking du Lac Brome vend lui aussi des unités de copropriété dans un but de location.

À priori, toutes ces données recueillies par Les Affaires ont de quoi nous réjouir car elles sont le reflet d'une grande confiance associée à la qualité du milieu naturel estrien. Par contre, on peut se demander si cette nouvelle mode du "condo de villégiature" n'est pas un peu trop euphorique et débridée. Tout cela est-il bien mesuré, rationnel? Un peu partout dans l'ouest de l'Estrie, on construit un grand nombre d'unités en copropriété, de 100,150 ou $200000 \$$ mais on ne développe pas parallèlement de volet activité - loisirattrait. On peut se demander alors qui louent ces luxueux "condos" et quel est le taux d'occupation des unités opérationnelles. Il y aura une bonne étude à faire sur ces facettes avant de tapisser complètement la région de ces immeubles de pacha. Surtout que les promoteurs ne jouissent pas tous d'une expertise mettant ie consommateur à l'abri de for désagréables surprises. En effet, une conclusion de la réputée firme de consultants Laventhol et Horwath qui a étudié ce dossier en 1986 peut nous inciter à la prudence: "En résumé, les projets que nous avons visités nous ont permis de constater un manque d'expérience des promoteurs, un optimisme exagéré, une absence de raffinement et une gestion négligée des projets" (31).
- la place - où tout Montréalais financièrement à l'aise doit avoir sa résidence secondaire. La demande actuelle ne témoigne pas du tout d'un ralentissement. Les prix de résidences secondaires qui se vendent le plus rapidement se situent entre $200000 \$$ et $300000 \$$. Plus encore, le prix des terrains sur les rives du lac Memphrémagog n'ont pratiquement pas de prix (sic!). En fait, c'est devenu une mode. Et être à la mode, ça se paie. L'acheteur devra payer de $1500 \$$ à $2200 \$$ le pied de façade sur le lac! Les terrains d'un acre en bordure du lac peuven aisément dépasser les $5 \$$ le pied carré" "

Le centre de villégiature Chéribourg compte déja 300 villas offertes en huit modèles. Une vingtaine d'autres seront mises en chantier à l'automne 1988 et se vendront environ $100000 \$$ meublées. De son côté, la station 
TABLEAU 5

Dépenses de séjour selon les moyens d'hebergement utilisés par les touristes québécois tous buts de woyage, 1986

\begin{tabular}{|c|c|c|c|c|}
\hline $\begin{array}{l}\text { Modes } \\
\text { d'hébergement }\end{array}$ & $\begin{array}{l}\text { Voyages- } \\
\text { personnes } \\
\text { ('000) }\end{array}$ & $\begin{array}{l}\text { Nuitées } \\
\text { (c000) }\end{array}$ & $\begin{array}{l}\text { Dépenses } \\
\text { totales } \\
\text { (M\$) }\end{array}$ & $\begin{array}{l}\text { Dépenses } \\
\text { séjour } \\
\text { (\$) }\end{array}$ \\
\hline $\begin{array}{l}\text { Hottel } \\
\text { Motel } \\
\text { Chalet commercial }\end{array}$ & $\begin{array}{r}1391 \\
1213 \\
314 \\
\end{array}$ & $\begin{array}{l}3170 \\
3220 \\
1263 \\
\end{array}$ & $\begin{array}{r}310,8 \\
235,3 \\
48,2 \\
\end{array}$ & $\begin{array}{l}223 \\
194 \\
153\end{array}$ \\
\hline $\begin{array}{l}\text { Sous-total } \\
\text { hótellerie }\end{array}$ & 2918 & 7653 & 594,3 & 204 \\
\hline $\begin{array}{l}\text { Camping } \\
\text { Résidences } \\
\text { parents/amis } \\
\text { Chalet privé } \\
\text { Autre } \\
\text { Non spécifié }\end{array}$ & $\begin{array}{r}679 \\
8563 \\
2651 \\
394 \\
139 \\
\end{array}$ & $\begin{array}{r}2845 \\
23167 \\
7027 \\
1719 \\
217 \\
\end{array}$ & $\begin{array}{r}62,5 \\
502,2 \\
78,3 \\
64,7 \\
9,7 \\
\end{array}$ & $\begin{array}{r}92 \\
59 \\
29 \\
164 \\
70 \\
\end{array}$ \\
\hline $\begin{array}{l}\text { Tous modes } \\
\text { d'hébergement } \\
\text { confondus }\end{array}$ & 14882 & 40311 & 1221,0 & 82 \\
\hline
\end{tabular}

Source: Enquete des voyages des Canadiens, 1986, Statistiques canada

Tableau préparé par Jacques Luneau, Analyse et développement, ministère du Tourisme, Québec. Compilation speciale.

\section{Conclusion}

Au tout début de notre texte, nous prétendions que la villégiature en chalet privé était la mal-aimée de l'industrie touristique québécoise. Nous croyons peut-être avoir trouvé une explication à cette situation. Poursuivant une réflexion parallèle à la nôtre, notre camarade Jacques Luneau, du ministère du Tourisme, nous produit le tableau des "dépenses de séjour selon les moyens d'hébergement utilisés par les touristes québécois en 1986". Or ce tableau 5 est très instructif...

Si on consulte les données utilisées par le Ministère, les dépenses totales de séjour au chalet privé en 1986 , ont atteint $78.3 \$$ millions au Québec alors que de notre côté (corroboré par plusieurs autres études), nous évaluons son impact économique à... $850 \$$ millions annuellement, soit 11 fois plus que le ministère du Tourisme. Toujours selon les données du Ministère, les dépenses par sćjour au chalet se situe à... 29 \$ par voyagepersonne; il en coutterait donc trois fois plus cher pour un sejour en camping par rapport à un séjour en villégiature; de même, il en coûterait 2 fois plus cher pour un séjour chez des parents et amis qu'en villégiature!... Cela est-il plausible?

À notre avis, ce qui cause la confusion dans ce tableau, c'est l'approche de "dépenses directes". Cette approche fausse sérieusement les données dans le cas du séjour en chalet privé parce qu'elle ne peut se comparer avec la "dépense directe" en hébergement commercial. Voyons un cas concret: Jean-Paul et Ginette sont allés à leur chalet de Sutton en fin de semaine. L'essence pour l'auto, la bouffe et une petite visite au musée leur revient à 29 s chacun qu'ils ont sorti "directement" de leurs poches pour leur subsistance immédiate. De leur côté, Réal et Céline ont passé la mểme fin de semaine, mais à l'hốtel de Bromont. Quant ils ont quitté, ils ont acquitté une facture de $223 \$$.
Qu'est-ce qui explique une telle différence? Quant l'hôtelier vous remet une facture de 2235, pour le client c'est une dépense directe mais l'administration y a comptabilisé toutes ses dépenses indirectes; en effet, cette facture contribue à payer le chef cuisinier, les assurances sur l'établissement, l'amortissement sur l'immeuble, les coûts de chauffage, le nouvel ameublement et la peinture du balcon et les fleurs qui ornent le jardin, les mauvaises créances d'il $y$ a deux ans et la publicité du Boston Globe, etc... etc... Toutes ces dépenses, normales et fort justifiables, sont incluses dans la facture de Réal et Céline, mème s'ils ne le savent pas ou n'y pensent pas. Le villégiateur doit assumer lui aussi toutes ces dépenses-là mais la comptabilisation qu'on retrouve au tableau $5 \mathrm{n}^{7}$ en tient absolument pas compte. C'est pourquoi on ne peut comparer le $29 \$$ du chalet privé avec le $92 \$$ du camping ou le $223 \$$ de l'hôtel. La dépense attribuée au chalet privé est très nettement sous-évaluée dans ce cas; pour la situer dans le même contexte que les autres modes d'hébergement, il faut donc tenir compte de la dépense indirecte. À ce moment là, la villégiature sera peut-être un peu plus considérée par le ministère du Tourisme comme composante majeure de notre industrie. $f$

\section{Notes explicative:}

(1) ST-AMOUR, J.P. (1979) - La villégiature au Québee. pp. 23-24; MLCP (18g3); La place de la villegiature privée dans les loisirs des Ousbbeois et dans leur vie sociale et deonomique. Quebec, D. 5.

(2) BURNET, Louis (1963): Villegiature et tourisme sur les eótes de France. p. 9.

(3) RACINE, J. Bemard (1967): Le tourisme de wiWegiature en milieu rural, in Bulletin de I'Association des Geographes de l'Amérique française. pp. 159-169.

(4) NADEAU, Roger (1971); Le tourisme de villegiature dans l'Estrie; impact des résidences secondaires sur la vie économique, sociale et culturelle des Cantons de l'Est, p. 2.
(5) Par exemple, au début de 1988, r'Auberge Chénibourg dans la rógion de Magog-Orford devenait le Centre de vihegiature Choribourg

(6) COPPOCK, I.T, $(1976)$; Second Homes: Curse or Bilessing?. New York, Ed. Pergamon Press, pp. 2.3 .

7) Congress Library (1969); Second Home in the United States. Washington, p. 17.

(8) Statistique Canada (1972): Dietionnaire des termes du recensement, p. 47

9) BARBIER, R, (1967); Logements de vacances ef residences sccondaines dans le Sud-Est medier. raneien, in Les Cahiers du Tourisme Súrie A, Cahier 5, no 196, Aix-en-Provence.

[10) GILEERT, Danielle (1985); La villógiature privêe: application aै la région do Magog-Orford, theses de maitrise, Departement de gutographie, Univer. site de Sherbrooke, p. 16.

(11) GILBERT, Danielle et NADEAU, Roger (1996); La villegiature privede au Qubbec: example de la rägion de Magog-Orford. Bulletin de recherche nos 90-91, Departement de gégraphie, Université de Sherbrooke, p. 65 .

(12) Catte étude subventionnéte par la Socidtó Canadienne d'Hypotheques et de Logement a conduit a la publication des ouvrages cites en (10) et 111).

(13) NADEAU, Roger (1971); op. cit. (4).

(14) NADEAU 11971) obtenait un taux de $29.7 \%$.

-Le Muskoka Lakes Association, en Ontario allait chercher un taux de $36 \%$ pour le même genre d'enquolte en 1984 ltire du Toronto Star, sept. $1994)$.

- Marcel Samson a obtenu un taux net de $42,6 \%$ pour un sondage sur les résidences secondaires dewenues permanentes. Contre-urbanisation ef résidences secondaires, in TÁoros, vol. 3, no 3, octobre 1983, pp, 13-17,

(15) Organisation Mondiale du Tourisme (1976); Manuel sur la móthodologie des tetudes de mar. ché, Madrid, 172 pages.

(16) TFR $=$ Nombre de résidences secondaires $\times 100$ Nombre de résidences principales in BAFBIEA (1967); op. eit. (9).

(17) Les données recueillies dans le róle d'dvaluation par Nadeau datent de 1968 alors que l'enquête aupress des proprietaires a śté réalisAe en 1971 (4).

(18) Aucun proprietaire de la région avait moins de 25 ans lors de notre enquete en 1984. La movenne d'äge québeccoise a êté calculée Aa partír du recen. sement de Statistique Canada de 1881 .

(19) MLCP (1983); op. cit. (1).

(20) Etabli selon la classification des professions de Sta. tisstique Canada, 1990.

(21) ST-AMOUR, J.-P. (1979); op. cit. (1)

(22) (15 invités $\times 2$ jours de sefour) $x 4650$ chalets: 139 E00 jours/année/personne.

(23) (En 1984, 20\% des propriétaires louent leur chalet, ce qui représente 928 chalets disponibles pour des pedriodes plus ou moins longues de location.

(24) COPPOCK, J.T. (1976), op, cit. [6).

(25) CRIBIER. Françoise [1976): Les résidences secon. daires des citadins dans les campagnes hancaises, in Etudes rurales. No. 49-60, Paris, pp. 181-204.

(26) MUSKOKA LAKES ASS., 1984 (14)

(27) FAPEL (1982); La villógiature, une force économique, Montréal, 10 pages.

(29) MLCP (1098), op. cit. (1)

(29) MLCP (1983), op. ett., p. 5 (1)

(30) GAGNON, Gilles, GAGNE, Jean-Paul, Residences secondaires, in Les Affaires, vol. LX, no 14, Montréal, 2 avril 1988, pp. 4-5.

(31) LAVENTHOL \& HOPWATH, Analyse du phánomène de la copropriété de villéglature au Québac, étude rebalisese pour le ministêre du Tourisme du Ouébec, Montreal, avTil 1986, p. 62. 